Discrete Comput Geom 33:57-65 (2005)

DOI: $10.1007 / \mathrm{s} 00454-004-1126-2$

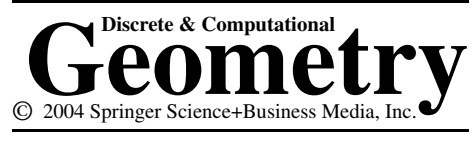

\title{
Valuations on Convex Sets of Oriented Hyperplanes
}

\author{
John Gates, ${ }^{1}$ Daniel Hug, ${ }^{2}$ and Rolf Schneider ${ }^{2}$ \\ ${ }^{1}$ Les Grezes, Villetoureix 24600, France \\ jp.gates@wanadoo.fr \\ ${ }^{2}$ Mathematisches Institut, Albert-Ludwigs-Universität, \\ D-79104 Freiburg i. Br., Germany \\ \{daniel.hug, rolf.schneider\}@ math.uni-freiburg.de
}

\begin{abstract}
We discuss valuations on convex sets of oriented hyperplanes in $\mathbb{R}^{d}$. For $d=2$, we prove an analogue of Hadwiger's characterization theorem for continuous, rigid motion invariant valuations.
\end{abstract}

\section{Introduction}

According to Hadwiger's characterization theorem, the only real functions on the space of convex bodies in $\mathbb{R}^{d}$ which are additive (valuations), continuous, and rigid motion invariant are the linear combinations of the intrinsic volumes. The surveys [25] and [24] show how this celebrated result is embedded in the theory of valuations on convex bodies. In recent years this theory has been enriched by various classification and characterization results for valuations under different assumptions, which were all inspired by Hadwiger's theorem; see [1]-[7], [12]-[23], and [26].

The present paper aims at a "dual" version of Hadwiger's theorem: instead of convex sets of points, we consider convex sets of oriented hyperplanes in $\mathbb{R}^{d}$. We formulate a conjectured analogue of Hadwiger's theorem, and we prove it for $d=2$. In that case the result says that a continuous, rigid motion invariant valuation on compact convex sets of oriented lines in the plane is a linear combination of three functions: the restriction of the Haar measure, the total angular measure of the directions, and the Euler characteristic.

\section{Convex Sets of Oriented Hyperplanes}

By $\mathbb{R}^{d}$ we denote $d$-dimensional real vector space $(d \geq 2)$ with scalar product $\langle\cdot, \cdot\rangle$. Its unit sphere is $S^{d-1}:=\left\{u \in \mathbb{R}^{d}:\langle u, u\rangle=1\right\}$. An oriented hyperplane in $\mathbb{R}^{d}$ 
consists of a $(d-1)$-dimensional affine subspace together with a normal vector giving its orientation. Thus, the oriented hyperplanes are in one-to-one correspondence with the pairs $(u, t) \in S^{d-1} \times \mathbb{R}$, where $\left\{x \in \mathbb{R}^{d}:\langle x, u\rangle=t\right\}$ is the unoriented hyperplane and $u$ gives the orientation. In this paper we write $H_{u, t}$ for the oriented hyperplane determined by $(u, t)$. We call $u$ the normal vector of $H_{u, t}$ and set $v\left(H_{u, t}\right):=u$, thus for a set $M$ of oriented hyperplanes, $v(M)$ is the set of all normal vectors of the elements of $M$. The set of oriented hyperplanes of $\mathbb{R}^{d}$ is denoted by $\mathcal{H}^{d}$.

The following representation is convenient. We identify $\mathbb{R}^{d}$ with the subspace of $\mathbb{R}^{d+1}$ spanned by the first $d$ vectors of the standard orthonormal basis, and we denote the remaining basis vector by $e$. Then the map $\gamma$ defined by

$$
\gamma\left(H_{u, t}\right):=u+t e
$$

maps the set $\mathcal{H}^{d}$ bijectively onto the cylinder $Z:=S^{d-1}+\mathbb{R} e$ in $\mathbb{R}^{d+1}$. The usual topology on $\mathcal{H}^{d}$ is defined by requiring that $\gamma$ be a homeomorphism, where $Z$ has its standard topology. By $\mu$ we denote the Lebesgue measure on $Z$, that is, the image measure under the map $(u, t) \mapsto u+t e$ of the product measure of spherical Lebesgue measure on $S^{d-1}$ and Lebesgue measure on $\mathbb{R}$. It is well known that the image measure of $\mu$ under $\gamma^{-1}$ is invariant under rigid motions and thus is the Haar measure of $\mathcal{H}^{d}$, with a special normalization.

By $\pi: \mathbb{R}^{d+1} \rightarrow \mathbb{R}^{d}$ we denote the orthogonal projection.

A definition of convexity, for sets of directed lines in the plane, was put forward in [9], and convex hulls of sets of oriented planes in $\mathbb{R}^{3}$ were considered in [10]. We give here the general definition for $\mathbb{R}^{d}$ and then restrict ourselves to a smaller class of convex sets. For an oriented hyperplane $H$, let $H^{+}, H^{-}$denote the positive and negative closed halfspace bounded by $H$ (i.e., $H^{+}=\left\{x \in \mathbb{R}^{d}:\langle x, u\rangle \geq t\right\}$ and $H^{-}=\left\{x \in \mathbb{R}^{d}:\langle x, u\rangle \leq t\right\}$ if $\left.H=H_{u, t}\right)$. For a set $M \subset \mathcal{H}^{d}$ put $P(M):=\bigcap_{H \in M} H^{+}$and $N(M):=\bigcap_{H \in M} H^{-}$. For an ordered pair $(P, N)$ of subsets of $\mathbb{R}^{d}$ define $E(P, N):=\left\{H \in \mathcal{H}^{d}: P \subset H^{+}, N \subset\right.$ $\left.H^{-}\right\}$. Then, by definition, the convex hull of a set $M \subset \mathcal{H}^{d}$ is $[M]:=E(P(M), N(M))$, and $M$ is convex if $[M]=M$.

We relate this to segments. Let $H_{u, t}$ and $H_{v, s}$ be oriented hyperplanes. The segment joining $H_{u, t}$ and $H_{v, s}$ is defined by

$\left[H_{u, t}, H_{v, s}\right]:=\left\{H_{w, p}: w=\frac{(1-\lambda) u+\lambda v}{\|(1-\lambda) u+\lambda v\|}, p=\frac{(1-\lambda) t+\lambda s}{\|(1-\lambda) u+\lambda v\|}, 0 \leq \lambda \leq 1\right\}$,

with the parameter value $\lambda=\frac{1}{2}$ excluded if $v=-u$. One can check that $\left[H_{u, t}, H_{v, s}\right]=$ $\left[\left\{H_{u, t}, H_{v, s}\right\}\right]$. In particular, $\left[H_{u, t}, H_{u, s}\right]$ is the set of all oriented hyperplanes with the same normal vector $u$ and lying between $H_{u, t}$ and $H_{u, s}$. If $u \neq \pm v$, then the segment $\left[H_{u, t}, H_{v, s}\right]$ consists of all oriented hyperplanes containing the intersection of both hyperplanes and having a normal vector in the positive hull of $u$ and $v$. Oriented hyperplanes $H_{u, t}$ and $H_{-u, s}$ are called antipodal. Note that segments joining antipodal hyperplanes have a special character: $\left[H_{u, t}, H_{-u,-t}\right]=\left\{H_{u, t}, H_{-u,-t}\right\}$ is not connected, and $\left[H_{u, t}, H_{-u, s}\right]$ with $s \neq-t$ is neither connected nor compact. The notion of a segment joining two oriented hyperplanes is invariant under affine transformations of $\mathbb{R}^{d}$ (this is not true for the parametrizations used in the definition). 
A set $M \subset \mathcal{H}^{d}$ is called segment-convex if with every pair of oriented hyperplanes it includes the segment joining them. The following result was proved in [9] for $d=2$; the proof extends to higher dimensions: if $M \subset \mathcal{H}^{d}$ is compact, then $M$ is convex if and only if it is segment-convex.

In studying valuations, it is convenient (and sufficient) to consider a restricted class of convex sets. By a convex body in $\mathcal{H}^{d}$ we understand a nonempty compact convex set in $\mathcal{H}^{d}$ which does not contain a pair of antipodal hyperplanes and is segment-convex. Thus a convex body is convex, and it is easy to see that every compact convex set is a finite union of convex bodies. The set of all convex bodies in $\mathcal{H}^{d}$ is denoted by $\mathcal{K}\left(\mathcal{H}^{d}\right)$.

With every set $M \subset \mathcal{H}^{d}$ we associate the cone in $\mathbb{R}^{d+1}$ defined by

$$
\Gamma(M):=\{\lambda \gamma(H): H \in M, \lambda \geq 0\} .
$$

Then a set $M \subset \mathcal{H}^{d}$ is segment-convex if and only if the cone $\Gamma(M)$ is convex in $\mathbb{R}^{d+1}$. Let $K \in \mathcal{K}\left(\mathcal{H}^{d}\right)$. Then $\Gamma(K) \cap \mathbb{R} e=\{0\}$. The projection $\pi(\Gamma(K))$ is a convex cone in $\mathbb{R}^{d}$ which is closed, as follows from the compactness of $\Gamma(K) \cap Z$, and is pointed, i.e., does not contain a line (since $K$ does not contain two antipodal hyperplanes). Hence, there exists a supporting hyperplane $S$ of this cone in $\mathbb{R}^{d}$ which meets the cone only at zero. It follows that the set $v(K)$ of normal vectors of the elements of $K$ is contained in some open hemisphere of $S^{d-1}$. Moreover, the hyperplane $S+\mathbb{R} e$ in $\mathbb{R}^{d+1}$ supports the cone $\Gamma(K)$ and meets it only at the origin. We denote by $\mathcal{K}(Z)$ the family of all sets of the form $C \cap Z$, where $C \neq\{0\}$ is a closed convex cone in $\mathbb{R}^{d+1}$ which has a supporting hyperplane containing $\mathbb{R} e$ and meeting $C$ only at the origin. If $K \in \mathcal{K}(Z)$, then $\gamma^{-1}(K) \in \mathcal{K}\left(\mathcal{H}^{d}\right)$. Thus, the convex bodies in $\mathcal{H}^{d}$ and the sets of $\mathcal{K}(Z)$ are in one-to-one correspondence.

Let $M \subset \mathcal{H}^{d}$ be a set of oriented hyperplanes whose normal vectors all lie in some open hemisphere. We define

$$
\operatorname{conv} M:=\gamma^{-1}(Z \cap \operatorname{pos} \gamma(M)),
$$

where pos denotes the positive hull in $\mathbb{R}^{d+1}$. If $M$ is compact, this coincides with [M], hence in this case conv $M$ is the convex hull of $M$. A polytope in $\mathcal{H}^{d}$ is the convex hull of a finite set of oriented hyperplanes whose normal vectors lie in some open hemisphere; it is a convex body in $\mathcal{H}^{d}$. A polytope in $\mathcal{H}^{2}$ is called a polygon.

We use the Hausdorff metric on compact subsets of $\mathbb{R}^{d+1}$ to topologize $\mathcal{K}(Z)$, and we use the map $\gamma^{-1}$ to carry this topology over to $\mathcal{K}\left(\mathcal{H}^{d}\right)$. Note that the resulting topology on $\mathcal{K}\left(\mathcal{H}^{d}\right)$ is invariant under rigid motions, although the Hausdorff metric, transferred via $\gamma^{-1}$, would not have this property.

The polytopes in $\mathcal{H}^{d}$ are dense in $\mathcal{K}\left(\mathcal{H}^{d}\right)$. This can be deduced from the corresponding result in Euclidean convexity. If $K \in \mathcal{K}\left(\mathcal{H}^{d}\right)$ is given, we can choose a hyperplane $S$ in $\mathbb{R}^{d+1}$, not through the origin, that intersects the cone $\Gamma(K)$ in a nonempty compact convex set. This set can be approximated by a sequence $\left(P_{i}\right)_{i \in \mathbb{N}}$ of convex polytopes in $S$. Almost all of the sets $\gamma^{-1}\left(Z \cap \operatorname{pos} P_{i}\right)$ are polytopes in $\mathcal{H}^{d}$, and they approximate $K$.

The set $\mathcal{H}_{x}$ of all oriented hyperplanes in $\mathcal{H}^{d}$ containing some fixed point $x \in \mathbb{R}^{d}$ is called a bundle. Since $H_{u, t} \in \mathcal{H}_{x}$ is equivalent to $\langle u+t e, x-e\rangle=0$, the cone $\Gamma\left(\mathcal{H}_{x}\right)$ is the hyperplane through the origin in $\mathbb{R}^{d+1}$ which is orthogonal to the vector $x-e$. 
The set $\mathcal{H}_{L}$ of all oriented hyperplanes in $\mathcal{H}^{d}$ whose normal vectors are contained in some $(d-1)$-dimensional linear subspace $L$ of $\mathbb{R}^{d}$ is called a pencil. If $L=x^{\perp}$ with $x \in \mathbb{R}^{d} \backslash\{0\}$, then $H_{u, t} \in \mathcal{H}_{L}$ is equivalent to $\langle u, x\rangle=0$, hence the cone $\Gamma\left(\mathcal{H}_{L}\right)$ is contained in the hyperplane through the origin in $\mathbb{R}^{d+1}$ which is orthogonal to the vector $x$. A convex body in $\mathcal{H}^{d}$ which is contained either in some bundle or in some pencil is called degenerate.

\section{Valuations on $\mathcal{K}\left(\mathcal{H}^{d}\right)$}

Let $S$ be a set and let $\mathcal{S}$ be a class of subsets of $S$ which is intersectional, that is, satisfies $A \cap B \in \mathcal{S}$ if $A, B \in \mathcal{S}$. A function $\varphi: \mathcal{S} \rightarrow \mathbb{R}$ is called additive or a valuation if $\varphi(A \cup B)+\varphi(A \cap B)=\varphi(A)+\varphi(B)$ whenever $A, B, A \cup B \in \mathcal{S}$. One also assumes that $\varphi(\emptyset)=0$ if $\emptyset \in \mathcal{S}$.

We are interested in valuations on $\mathcal{K}\left(\mathcal{H}^{d}\right)$, the set of convex bodies in the space $\mathcal{H}^{d}$ of oriented hyperplanes in $\mathbb{R}^{d}$. First we remark that our restriction to convex bodies in $\mathcal{H}^{d}$ is not an essential restriction in this context: every continuous valuation on $\mathcal{K}\left(\mathcal{H}^{d}\right)$ has an additive extension to the set of finite unions of elements from $\mathcal{K}\left(\mathcal{H}^{d}\right)$. This can be shown by adapting Groemer's [11] proof to the present situation, working with the convex cones $\Gamma(K), K \in \mathcal{K}\left(\mathcal{H}^{d}\right)$, and with hyperplanes through the origin in $\mathbb{R}^{d+1}$.

We ask particularly for valuations on $\mathcal{K}\left(\mathcal{H}^{d}\right)$ which are continuous and invariant under rigid motions, in analogy to the assumptions of Hadwiger's characterization theorem. First we give examples. The immediate one is given by the restriction of the Haar measure, denoted by $\eta$, thus $\eta(K)=\mu(\gamma(K))$ for $K \in \mathcal{K}\left(\mathcal{H}^{d}\right)$. It is simple. Here, a valuation on $\mathcal{K}\left(\mathcal{H}^{d}\right)$ is called simple if it vanishes on degenerate convex bodies in $\mathcal{H}^{d}$. The continuity of $\eta$ on $\mathcal{K}\left(\mathcal{H}^{d}\right)$ can be proved as follows. For $K \in \mathcal{K}\left(\mathcal{H}^{d}\right)$, let

$$
\bar{\Gamma}(K):=\{\lambda \gamma(H): H \in K, 0 \leq \lambda \leq 1\} .
$$

If $V$ denotes the volume in $\mathbb{R}^{d+1}$, it is easy to see that

$$
V(\bar{\Gamma}(K))=\frac{1}{d+1} \mu(\gamma(K))=\frac{1}{d+1} \eta(K) .
$$

Since the map $K \mapsto \bar{\Gamma}(K)$ is continuous, the continuity of $\eta$ now follows from the known continuity of the volume on the space of convex bodies in $\mathbb{R}^{d+1}$.

A trivial example of a continuous, motion invariant valuation on $\mathcal{K}\left(\mathcal{H}^{d}\right)$ is given by a constant function. The function $\chi$ with $\chi(K)=1$ for $K \in \mathcal{K}\left(\mathcal{H}^{d}\right)$ is called the Euler characteristic, for the reason that the Euler characteristic known from topology is additive and attains the value one on convex bodies.

Further examples of valuations with the required properties are obtained as follows. By a convex body in the sphere $S^{d-1}$ we understand a nonempty set in $S^{d-1}$ which is the intersection of $S^{d-1}$ with a closed pointed convex cone in $\mathbb{R}^{d}$. Equivalently, this is a nonempty closed subset of $S^{d-1}$ which lies in some open hemisphere and is geodesically convex. By $\mathcal{K}\left(S^{d-1}\right)$ we denote the set of all convex bodies in $S^{d-1}$.

Let $\psi$ be a valuation on $\mathcal{K}\left(S^{d-1}\right)$. Then

$$
\varphi(K):=\psi(v(K)), \quad K \in \mathcal{K}\left(\mathcal{H}^{d}\right),
$$


defines a valuation $\varphi$ on $\mathcal{K}\left(\mathcal{H}^{d}\right)$ (observe that $v(K)$ is a convex body in $S^{d-1}$, by the consideration above). To prove the valuation property of $\varphi$, let $K_{1}, K_{2} \in \mathcal{K}\left(\mathcal{H}^{d}\right)$ be such that $K_{1} \cup K_{2} \in \mathcal{K}\left(\mathcal{H}^{d}\right)$. The relations $v\left(K_{1} \cup K_{2}\right)=v\left(K_{1}\right) \cup v\left(K_{2}\right)$ and $v\left(K_{1} \cap K_{2}\right) \subset$ $v\left(K_{1}\right) \cap v\left(K_{2}\right)$ are trivial. Let $u \in v\left(K_{1}\right) \cap v\left(K_{2}\right)$. There are oriented hyperplanes $H_{u, t} \in K_{1}$ and $H_{u, s} \in K_{2}$. Since $K_{1} \cup K_{2}$ is convex, the segment $\left[H_{u, t}, H_{u, s}\right.$ ] belongs to $K_{1} \cup K_{2}$. Since $K_{1}, K_{2}$ are closed, there exists a value $p$ between $t$ and $s$ for which $H_{u, p} \in K_{1} \cap K_{2}$, hence $u \in v\left(K_{1} \cap K_{2}\right)$. Now the additivity of $\varphi$ follows from $\varphi\left(K_{1} \cup\right.$ $\left.K_{2}\right)+\varphi\left(K_{1} \cap K_{2}\right)=\psi\left(v\left(K_{1} \cup K_{2}\right)\right)+\psi\left(v\left(K_{1} \cap K_{2}\right)\right)=\psi\left(v\left(K_{1}\right) \cup v\left(K_{2}\right)\right)+$ $\psi\left(v\left(K_{1}\right) \cap v\left(K_{2}\right)\right)=\psi\left(v\left(K_{1}\right)\right)+\psi\left(v\left(K_{2}\right)\right)=\varphi\left(K_{1}\right)+\varphi\left(K_{2}\right)$. If $\psi$ is continuous, then $\varphi$ is continuous. If $\psi$ is invariant under rotations, then $\varphi$ is invariant under rigid motions.

Examples of rotation invariant, continuous valuations on $\mathcal{K}\left(S^{d-1}\right)$ are given by the spherical intrinsic volumes $v_{0}, \ldots, v_{d-1}$. They can be defined as the coefficients in the spherical Steiner formula

$$
\sigma_{d-1}\left(K_{\varepsilon}\right)=\beta_{d-1} v_{d-1}(K)+\sum_{j=0}^{d-2} f_{j}(\varepsilon) \beta_{j} \beta_{d-2-j} v_{j}(K), \quad 0 \leq \varepsilon \leq \pi / 2 .
$$

Here, $\sigma_{d-1}$ denotes spherical Lebesgue measure on $S^{d-1}$. For $K \in \mathcal{K}\left(S^{d-1}\right)$ and $0 \leq$ $\varepsilon \leq \pi / 2$, the set $K_{\varepsilon}$ is the set of all points of $S^{d-1}$ with spherical distance from $K$ at most $\varepsilon$. Finally, $\beta_{j}:=\sigma_{j}\left(S^{j}\right)$, and

$$
f_{j}(\varepsilon):=\int_{0}^{\varepsilon} \cos ^{j} t \sin ^{d-2-j} t d t .
$$

For references to treatments of the properties of the spherical intrinsic volumes and to the role they play in spherical integral geometry, we refer to [8].

The following theorem provides some information about the structure of rigid motion invariant, continuous valuations on $\mathcal{K}\left(\mathcal{H}^{d}\right)$.

Theorem 1. Every continuous, rigid motion invariant valuation $\varphi$ on $\mathcal{K}\left(\mathcal{H}^{d}\right)$ has a unique representation of the form

$$
\varphi(K)=\varphi^{\prime}(K)+\psi(v(K)), \quad K \in \mathcal{K}\left(\mathcal{H}^{d}\right),
$$

where $\varphi^{\prime}$ is a simple, continuous, rigid motion invariant valuation on $\mathcal{K}\left(\mathcal{H}^{d}\right)$ and $\psi$ is a continuous, rotation invariant valuation on $\mathcal{K}\left(S^{d-1}\right)$.

Proof. For $M \in \mathcal{K}\left(S^{d-1}\right)$ we set

$$
\langle M\rangle:=\left\{H_{u, 0}: u \in M\right\} .
$$

Then $\langle M\rangle \in \mathcal{K}\left(\mathcal{H}^{d}\right)$, hence we can define

$$
\psi(M):=\varphi(\langle M\rangle) .
$$

Clearly, $\psi$ is a continuous, rotation invariant valuation on $\mathcal{K}\left(S^{d-1}\right)$. Put

$$
\varphi^{\prime}(K):=\varphi(K)-\psi(\nu(K)) \quad \text { for } \quad K \in \mathcal{K}\left(\mathcal{H}^{d}\right) .
$$

Then $\varphi^{\prime}$ is a continuous, rigid motion invariant valuation on $\mathcal{K}\left(\mathcal{H}^{d}\right)$. 
Let $K \in \mathcal{K}\left(\mathcal{H}^{d}\right)$ be degenerate. Suppose, first, that $K$ is contained in some bundle $\mathcal{H}_{x}$. The translation by the vector $-x$ carries $K$ into $\langle v(K)\rangle$, hence $\varphi(K)=\varphi(\langle v(K)\rangle)=$ $\psi(v(K))$, thus $\varphi^{\prime}(K)=0$. Assume, next, that $K$ is contained in some pencil. It is easy to see, by applying suitable rotations in $\mathbb{R}^{d+1}$ to the cone $\Gamma(K)$, that $K$ can be approximated by degenerate convex bodies contained in bundles. Continuity now implies that $\varphi^{\prime}(K)=0$. This shows that $\varphi^{\prime}$ is simple. The uniqueness of $\psi$ and $\varphi^{\prime}$ is clear. This completes the proof.

Now it is time to formulate a conjecture.

Conjecture. Every continuous, rigid motion invariant valuation $\varphi$ on $\mathcal{K}\left(\mathcal{H}^{d}\right)$ is of the form

$$
\varphi(K)=c_{d} \eta(K)+\sum_{j=0}^{d-1} c_{j} v_{j}(\nu(K)), \quad K \in \mathcal{K}\left(\mathcal{H}^{d}\right),
$$

with constants $c_{0}, \ldots, c_{d}$.

Theorem 1 shows that the truth of this conjecture would follow from proofs of the following two conjectures: (a) every simple, continuous, rigid motion invariant valuation on $\mathcal{K}\left(\mathcal{H}^{d}\right)$ is a constant multiple of the Haar measure $\eta$; (b) every continuous, rotation invariant valuation on $\mathcal{K}\left(S^{d-1}\right)$ is a linear combination of the spherical intrinsic volumes $v_{0}, \ldots, v_{d-1}$. Conjecture (b) has been formulated repeatedly; see [8] for references (and Chapter 11 of [16] for a proof in the case $d=3$ ). Its truth would follow (by induction with respect to the dimension) from a proof of the following conjecture: (c) every simple, continuous, rotation invariant valuation on $\mathcal{K}\left(S^{d-1}\right)$ is a constant multiple of the spherical volume. Probably conjectures (c) and (a) are of comparable difficulty.

\section{Proof of the Conjecture for $d=2$}

We prove the conjecture for $d=2$. Let $\varphi$ be a continuous, rigid motion invariant valuation on $\mathcal{K}\left(\mathcal{H}^{2}\right)$. Let $\varphi^{\prime}$ and $\psi$ be defined according to Theorem 1; in particular, $\psi(M)=\varphi(\langle M\rangle)$ for $M \in \mathcal{K}\left(S^{1}\right)$. On one-pointed sets in $S^{1}$, the rotation invariant valuation $\psi$ attains a constant value $c_{0}$, and $\psi-c_{0} \chi$ is a continuous, rotation invariant valuation on $\mathcal{K}\left(S^{1}\right)$ which is simple, that is, vanishes on one-pointed sets. Here, $\chi$ denotes the Euler characteristic, that is, the valuation on $\mathcal{K}\left(S^{1}\right)$ satisfying $\chi(M)=1\left(=2 v_{0}(M)\right)$ for $M \in \mathcal{K}\left(S^{1}\right)$. By Proposition 11.2.2 of [16], the simple valuation $\psi-c_{0} \chi$ on $\mathcal{K}\left(S^{1}\right)$ is a constant multiple of the length $\sigma_{1}$, which is equal to $v_{1} / 2 \pi$. Since $\chi(\nu(K))=1=\chi(K)$ for $K \in \mathcal{K}\left(\mathcal{H}^{2}\right)$, we thus get $\psi(v(K))=c_{0} \chi(K)+c_{1} \sigma_{1}(v(K))$. For $K \in \mathcal{K}\left(\mathcal{H}^{2}\right)$, we call $\sigma_{1}(v(K))$ the angular range of $K$.

Our main task is to show that $\varphi^{\prime}$ is a constant multiple of the Haar measure $\eta$. For this, we use vertical triangles. A vertical triangle $T$ is defined as the convex hull of three oriented lines $H_{u, t}, H_{u, s}$, and $H_{v, r}$ with $v \neq \pm u$. On the cylinder $Z$, the points $\gamma\left(H_{u, t}\right)$ and $\gamma\left(H_{u, s}\right)$ are on the same generator, which is the reason for the term "vertical." We 
call $h=|t-s|$ the height of $T$. The angle $\theta$ of $T$ is defined by requiring that $|\theta| \in(0, \pi)$ is the angle between $u$ and $v$ and that $\theta>0$ if and only if the pair $(u, v)$ is positively oriented (with respect to the standard orientation of $\mathbb{R}^{2}$ ); in the latter case we also say that $\theta$ is the angle from $u$ to $v$. First we compute $\eta(T)$. We may assume that $\theta>0$. An oriented line $H_{w, p}$ belongs to the vertical triangle $T$ if and only if $w \in \operatorname{pos}\{u, v\}$ and $p$ is contained in a certain interval of length $h[\cos \alpha-\cot \theta \sin \alpha]$, where $\alpha$ is the angle from $u$ to $w$. Hence,

$$
\eta(T)=\int_{0}^{\theta} h[\cos \alpha-\cot \theta \sin \alpha] d \alpha=h \tan (\theta / 2) .
$$

In general,

$$
\eta(T)=h \tan |\theta / 2| .
$$

Let $T(h, \theta)$ be a vertical triangle with height $h$ and angle $\theta$. By the rigid motion invariance of $\varphi^{\prime}$, the value $\varphi^{\prime}(T(h, \theta))$ depends only on $h$ and $\theta$, so that we can define $\varphi^{\prime}(T(h, \theta))=: F(h, \theta)$. A vertical triangle $T(h+k, \theta)$ can be decomposed into two vertical triangles with heights $h$ and $k$, respectively, and angle $\theta$. Here "decomposition" is meant to imply that the intersection of the components is a degenerate convex body. Since $\varphi^{\prime}$ is simple, we infer that $F(h+k, \theta)=F(h, \theta)+F(k, \theta)$, for arbitrary $h, k \geq 0$. By continuity, this implies that

$$
F(h, \theta)=h f(\theta)
$$

with some function $f$, which is continuous, too.

Let $0<\theta<\pi$ and $0<\omega<\pi$ with $\theta+\omega<\pi$ be given. We choose oriented lines $g_{1}=H_{u, 0}, g_{2}=H_{u, 1}, g_{3}=H_{v, 0}$, and $g_{4}=H_{w, 0}$, such that the angle from $u$ to $v$ is equal to $\theta$ and the angle from $v$ to $w$ is equal to $\omega$. Let $g_{5}=H_{v, r}$ be the oriented line parallel to $g_{3}$ through the intersection point of $g_{2}$ and $g_{4}$. It is easy to check (considering the images $\gamma\left(g_{i}\right)$ on the cylinder $Z$ ) that the vertical triangle conv $\left\{g_{1}, g_{2}, g_{4}\right\}$ (of height 1 and angle $\theta+\omega)$ can be decomposed into the vertical triangles $\operatorname{conv}\left\{g_{3}, g_{4}, g_{5}\right\}$, of height

$$
h=\frac{\sin \omega}{\sin (\theta+\omega)}
$$

and angle $\omega, \operatorname{conv}\left\{g_{1}, g_{2}, g_{3}\right\}$ (of height 1 and angle $\theta$ ), and $\operatorname{conv}\left\{g_{2}, g_{3}, g_{5}\right\}$ (of height $h$ and angle $-\theta$ ). Since $\varphi^{\prime}$ is a simple valuation (and the union of the two latter vertical triangles is convex), it follows that

$$
F(1, \theta+\omega)=F(h, \omega)+F(1, \theta)+F(h,-\theta),
$$

hence

$$
f(\theta+\omega)=f(\theta)+\frac{\sin \omega}{\sin (\theta+\omega)}[f(-\theta)+f(\omega)] .
$$

First, we let $0<\alpha<\pi$ and choose $\theta=\omega=\alpha / 2$, to obtain

$$
f(\alpha)=f(\alpha / 2)\left[1+\frac{1}{2 \cos (\alpha / 2)}\right]+\frac{1}{2 \cos (\alpha / 2)} f(-\alpha / 2) .
$$


A similar relation holds with $\alpha$ replaced by $-\alpha$. By subtraction we get $f(\alpha)-f(-\alpha)=$ $f(\alpha / 2)-f(-\alpha / 2)$. Iterating this relation and observing that $f$ is continuous and $f(0)=$ 0 , we conclude that

$$
f(\alpha)=f(-\alpha) .
$$

Hence, (2) now reads

$$
f(\theta+\omega)=f(\theta)+\frac{\sin \omega}{\sin (\theta+\omega)}[f(\theta)+f(\omega)] .
$$

For $\theta \in(0, \pi)$, we obtain the right-hand derivative

$$
f_{r}^{\prime}(\theta)=\lim _{\omega \rightarrow 0+} \frac{f(\theta+\omega)-f(\theta)}{\omega}=\frac{1}{\sin \theta} f(\theta) .
$$

Replacing $\theta$ by $\theta-\omega$ in (4), we similarly get $f_{l}^{\prime}(\theta)=f(\theta) / \sin \theta$. Thus $f^{\prime}(\theta)=$ $f(\theta) / \sin \theta$, which implies $f(\theta)=c_{2} \tan (\theta / 2)$ for $0 \leq \theta<\pi$, with a constant $c_{2}$. In view of (1) and (3), this shows that

$$
\varphi^{\prime}(T)=c_{2} \eta(T)
$$

for every vertical triangle $T$.

Let $P$ be an arbitrary triangle in $\mathcal{H}^{2}$, that is, the convex hull of three oriented lines whose normal vectors lie in some semicircle. It is easy to see (e.g., using the representation on the cylinder $Z$ ) that $P$ can be decomposed into two vertical triangles, the intersection of which is a segment. It follows that $\varphi^{\prime}(P)=c_{2} \eta(P)$. Let $P$ be a polygon in $\mathcal{H}^{2}$. It can be decomposed into two polygons whose intersection is a segment and each of which has fewer vertices than $P$ (the vertices of $P$ are defined in the obvious way; they are in one-to-one correspondence with the extreme rays of the convex cone $\Gamma(P))$. Hence, we can prove by induction with respect to the number of vertices that $\varphi^{\prime}(P)=c_{2} \eta(P)$ holds for every polygon $P$ in $\mathcal{H}^{2}$. Since the polygons are dense in $\mathcal{K}\left(\mathcal{H}^{2}\right)$ and $\varphi^{\prime}$ is continuous, we conclude that $\varphi^{\prime}=c_{2} \eta$ on $\mathcal{K}\left(\mathcal{H}^{2}\right)$. Thus, we have proved the following theorem.

Theorem 2. Every continuous and rigid motion invariant valuation on the set $\mathcal{K}\left(\mathcal{H}^{2}\right)$ of convex bodies in the space of oriented lines in $\mathbb{R}^{2}$ is a linear combination, with constant coefficients, of the Haar measure, the angular range, and the Euler characteristic.

\section{References}

1. S. Alesker, Continuous rotation invariant valuations on convex sets, Ann. of Math. 149 (1999), 977-1005.

2. S. Alesker, Description of continuous isometry covariant valuations on convex sets, Geom. Dedicata 74 (1999), 241-248.

3. S. Alesker, On P. McMullen's conjecture on translation invariant valuations, Adv. Math. 155 (2000), 239263.

4. S. Alesker, Classification results on valuations on convex sets, in Progress in Mathematics, vol. 201, pp. 1-8, Birkhäuser, Basel, 2000.

5. S. Alesker, Description of translation invariant valuations on convex sets with solution of P. McMullen's conjecture, Geom. Funct. Anal. 11 (2001), 244-272. 
6. S. Alesker, Hard Lefschetz theorem for valuations, complex integral geometry, and unitarily invariant valuations, J. Differential Geom. 63 (2003), 63-95.

7. S. Alesker, SU(2)-invariant valuations, Preprint, 2003.

8. F. Gao, D. Hug, and R. Schneider, Intrinsic volumes and polar sets in spherical space, Math. Notae 41 (2001/02), $159-176$ (2003).

9. J. Gates, Some dual problems of geometric probability in the plane, Combin. Probab. Comput. 2 (1993), $11-23$.

10. J. Gates, Dual convexity in space and the Sylvester-Klee problem, Far East J. Math. Sci. 4 (2002), 1-20.

11. H. Groemer, On the extension of additive functionals on classes of convex sets, Pacific. J. Math. 75 (1978), 397-410.

12. D.A. Klain, A short proof of Hadwiger's characterization theorem, Mathematika 42 (1995), 329-339.

13. D.A. Klain, Star valuations and dual mixed volumes, Adv. Math. 121 (1996), 80-101.

14. D.A. Klain, Invariant valuations on star-shaped sets, Adv. Math. 125 (1997), 95-113.

15. D.A. Klain, Even valuations on convex bodies, Trans. Amer. Math. Soc. 352 (1999), 71-93.

16. D.A. Klain and G.-C. Rota, Introduction to Geometric Probability, Cambridge University Press, Cambridge, 1997.

17. M. Ludwig, A characterization of affine length and asymptotic approximation of convex discs, Abh. Math. Sem. Univ. Hamburg 69 (1999), 75-88.

18. M. Ludwig, Upper semicontinuous valuations on the space of convex discs, Geom. Dedicata 80 (2000), 263-279.

19. M. Ludwig, Valuations on polytopes containing the origin in their interiors, Adv. Math. 170 (2002), 239256.

20. M. Ludwig, Projection bodies and valuations, Adv. Math. 172 (2002), 158-168.

21. M. Ludwig, Moment vectors of polytopes, Rend. Circ. Mat. Palermo (2) Suppl. 70 (2002), vol. II, 123-138.

22. M. Ludwig, Ellipsoids and matrix valued valuations, Duke Math. J. 119 (2003), 159-188.

23. M. Ludwig and M. Reitzner, A characterization of affine surface area, Adv. Math. 147 (1999), 138-172.

24. P. McMullen, Valuations and dissections, in Handbook of Convex Geometry, vol. B (P.M. Gruber and J.M. Wills, eds.), pp. 933-988, Elsevier, Amsterdam, 1993.

25. P. McMullen and R. Schneider, Valuations on convex bodies, in Convexity and Its Applications (P.M. Gruber and J.M. Wills, eds.), pp. 170-247, Birkhäuser, Basel, 1983.

26. R. Schneider, Simple valuations on convex bodies, Mathematika 43 (1996), 32-39.

Received February 21, 2002, and in revised form January 7, 2004, and May 14, 2004.

Online publication October 20, 2004. 